\title{
Planning Author and Character Goals for Story Generation
}

\author{
Candice Jean Solis, Joan Tiffany Siy, Emerald Tabirao, and Ethel Ong \\ College of Computer Studies \\ De La Salle University \\ Manila, Philippines \\ candice_solis13@yahoo.com, emeraldtabirao_dlsulyahoo.com.ph, \\ joān.tiffany.siy@gmail.com, ethel.onḡ@delasalle.ph
}

\begin{abstract}
The design and content of the planning library of a story generation system dictates the content quality of the story it produces. This paper presents the story planner component of Picture Books, a system that generates stories for children aged 4 to 6 years based on a set of picture elements selected by the user. The planning library separates the design for the story patterns from the design of the semantic ontology that supplies the story's domain knowledge. An evaluation of the system shows that the coherency and completeness of the plot is attributed to the story pattern design structure while the appropriateness of the content is attributed to the semantic ontology.
\end{abstract}

\section{Introduction}

Several researchers have developed story generators capable of generating stories that closely resemble human-made stories. Some of these include TALE-SPIN (Meehan, 1977) that generates stories through problem solving, MINSTREL (Turner, 1992) that uses an episodic memory scheme for storing past problem-solving cases, and MAKEBELIEVE (Liu and Singh, 2002) that constructs stories with the use of logical reasoning.

Callaway and Lester (2002) observed that most story generators (SG), such as TALE-SPIN and MAKEBELIEVE, concentrated on the generation of plots and the characters, with less emphasis on linguistic phenomena, resulting in stories that have good narrative quality but lacking in linguistic structures. Their STORYBOOK system addresses this by applying full-scale linguistic approaches to the narrative prose generation architecture of AUTHOR (Callaway, 2000).

Loenneker (2005) made a similar observation regarding SG whose implicit goal is to generate a coherent narrative in a given genre with less emphasis on discourse structure, and skipping document structuring and microplanning (Reiter and Dale, 2000) stages.

In this paper, we present our story generator, Picture Books, which generates stories for children aged four to six. Picture Books derives the story elements from a given input set of picture elements (backgrounds, characters, and objects). The genre (fables) and story goal (moral lessons) are applied as separate parts of the system's planning library, which contains story planning goals to convert abstract story specifications to coherent stories suitable for the target age group.

The rest of this paper is subdivided as follows. Section 2 presents some background information on the specifications of a children's story as well as the semantic ontology used by the planners of Picture Books. Section 3 discusses the design of the plan library and the planning process involved in story generation, followed by the evaluation results on the content and grammar of the stories generated by the system in Section 4. The paper ends with a summary of future work that can be done to improve the generated stories.

\section{Storytelling and Picture Books}

The motivation behind Picture Books is twofold. First is the realization that stories are combinations of various genres to produce a differing effect. The genres can be considered as templates that dictate the plot of the story. It is therefore possible to create a story by indicating the story elements (i.e., characters, events, and settings), the genres, and the goal of the story to automatically produce a narrative text. The second motivation is that stories serve as rich sources of information that help develop a child's knowledge. However, young children recognize images of objects easily compared to words (Fields et al, 2003), accounting for the popularity of picture-based story books that allow readers to relate stories by using not only words, but pictures depicting the story. 


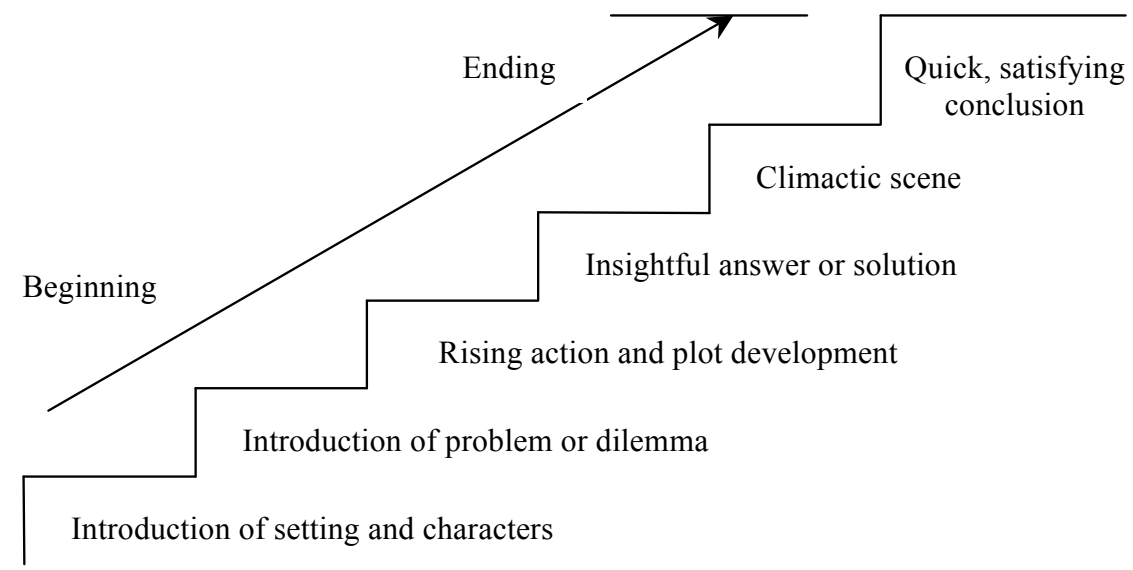

Figure 0. Common and Classic Story Pattern Form (Machado, 2003)

\subsection{Story Specifications}

Interviews with child educators revealed that most of the published storybooks for children focus on themes to teach them lessons about proper behavior. These themes revolve around everyday activities like eating on time and brushing your teeth with lessons on being careful, being honest, and the value of sharing. Themes also dictate the objects that can be used in the story, for example, in the eating healthy foods theme, possible positive objects supporting good behavior are apples and bananas, while cakes and candies are considered negative objects in cases of misbehavior (i.e., unhealthy foods).

Another common characteristic of children's stories is the use of the fable form, wherein the story characters are portrayed by animals that can capture the imagination and attention of the target readers. The animals have simple traits that children can relate with, such as loyalty for dogs, playfulness for cats, kindness for rabbits, and bravery for tigers. They are also given names, such as Ellen the elephant, Rizzy the rabbit, and Leo the lion, to give the impression that the characters are friends that the children are getting to know better through reading the story.

In the linguistic aspect, stories for four year olds have simple sentence structures and contain line redundancy. This is lessened as the child grows older. The words used in the stories are not only simple and easy to understand, but also vary depending on the child's age in order to introduce new words to his vocabulary. Lessons and rules are emphasized by positive praises while improper behaviors are emphasized by revealing their consequences.

Another aspect to consider in storytelling is the story's title. Story titles are short and often contain the story's theme as a hint to what the story is about. The main character's name should also be included in the title, such as "Leo the Lion Learns to Eat on Time".

In general, Machado (2003) showed that stories follow a common and classic story pattern depicted in Figure 0, which flows from negative to positive and has the following outline:

i. The main character wants something.

ii. The main character is informed of the rules and/or restrictions.

iii. The main character disobeys the rule.

iv. The main character is either caught or experiences natural consequences of disobedience (e.g. a tooth ache from eating too much candy).

v. The main character learns a lesson.

\subsection{Semantic Ontology}

The knowledge resource dictates the amount of information that an SG system can output, thus highlighting its importance. Picture Books uses an ontology to have a flexible knowledge resource that provides relevant concepts familiar to the target age group as well as applicable to the story being planned. Its design was adapted from ConceptNet (Liu and Singh, 2004a), a semantic resource with structure closely resembling that of WordNet (2006).

The nodes used by ConceptNet are of three general classes representing noun phrases, attributes, and activity phrases. A semantic relationship connects two concepts while a semantic category classifies them. The semantic relationships are binary relation types defined by Open Mind Commonsense project (Liu and Singh, 2004b). Table 1 lists some of these relationships defined in Picture Books following the form $<$ relationship $>(<$ concept $1>$, $<$ concept $2>)$. 


\begin{tabular}{|c|c|}
\hline $\begin{array}{l}\text { Semantic } \\
\text { Category }\end{array}$ & Semantic Relationships \\
\hline Things & $\begin{array}{l}\text { Is } \boldsymbol{A} \text { (headache, pain) - corresponds loosely } \\
\text { to hypernym in WordNet } \\
\text { PropertyOf(apple, healthy) } \\
\text { PartOf(window, pane) - corresponds } \\
\text { loosely to holonym in WordNet } \\
\text { MadeOf(toy car, clay) }\end{array}$ \\
\hline Events & $\begin{array}{l}\text { FirstSubeventOf(tell bedtime story, } \\
\text { sleep) } \\
\text { EventForGoalEvent(go to grocery store, } \\
\text { buy food) } \\
\text { EventForGoalState(clean up, be neat) } \\
\text { EventRequiresObject(play, toy) }\end{array}$ \\
\hline Actions & $\begin{array}{l}\text { EffectOf(become dirty, itchy) } \\
\text { EffectOfIsState(make friends, friendship) } \\
\text { CapableOf(toy car, play) }\end{array}$ \\
\hline
\end{tabular}

Table 1. ConceptNet semantic relationships (Liu and Singh, 2004b) with sample concepts of Picture Books

\section{Planning the Story}

Picture Books has three major components - a picture editor, a story planner, and a sentence planner. The Picture Editor is provided for users to specify the background or setting of the story (kitchen), and to select and "stick" into the background the set of characters (little sheep and mama sheep) and objects (cake, bread). An example picture is shown in Figure 2.

The first child character placed in the picture will be the protagonist of the story, while the first adult sticker placed in the picture will be the parent of the protagonist. If there is no adult character, the protagonist's biological parent will be chosen as the adult character needed in the story. The first object sticker placed in the picture will assume the \%object\% variable used in planning the story - especially in ontology accesses. The rest are discarded.

All elements in the picture, namely the background, the characters and the objects (including sequence of placement), and the name and age of the user, are stored in an input content representation (ICR) and passed on to the story planner.

The Story Planner takes in the abstract ICR then performs three planning steps - i) theme planning to select an appropriate theme for the story, ii) plot planning to instantiate the story plots depicting the theme, and iii) presentation planning to handle the planning of the story's title, introduction and appropriate ending. A Story Organizer arranges these resulting story events as it is supposed to be presented to the user in an abstract story tree.
The Sentence Planner then converts the abstract story tree representation to sentence specifications by performing referring expression generation, lexicalization, and phrase specification mapping. The Surface Realizer uses the simplenlg realiser (Venour and Reiter, 2008) to convert the sentence specifications to actual sentences that comprise the story.

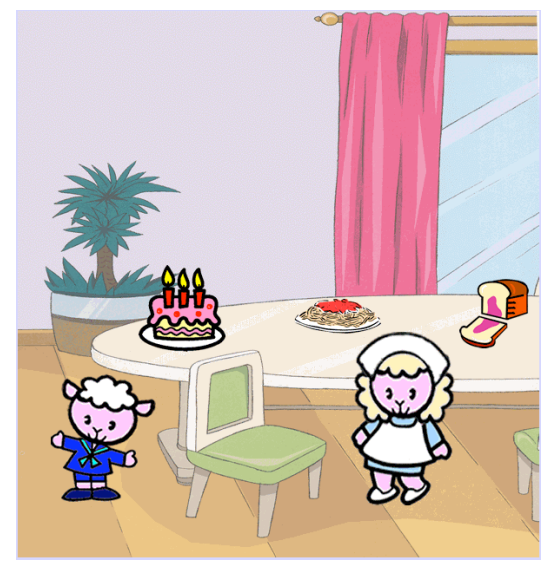

Figure 2. Sample Picture with Stickers

\subsection{Plan Library}

The plan library contains the set of instructions and information on creating the plot of a story, and has three parts: (1) the story elements containing information regarding the characters, objects and settings of a story; (2) a set of story patterns to direct the story goal towards attainment of the moral lesson; and (3) a semantic ontology containing concepts applicable to the target domain, in this case, fables.

Picture Books uses a set of predefined characters, objects and backgrounds, as inputs. These story elements are used to determine the actors, the setting and the theme of a story. Information on characters, such as the parents, is useful for identifying other characters in the story. The background serves as the main setting of the story, and combined with the selected objects, is used to determine the theme. Given a bedroom background, the set of available themes include being brave, being neat, being careful, being honest, and sleeping early. Objects that are available for this background include an alarm clock, a lamp, a pillow, and toys.

The story pattern is used to direct the goal of the story and is composed of the theme, story plot, author goal and character goal. Each component is designed to subsume the next (i.e., theme subsumes story plot, and story plot subsumes author goal) to support different granular- 
ity of story details and to lessen the redundancy as common story details are subsumed by a higher-level component.

A theme dictates the plot of a story and is composed of four story plots (see Figure 3) namely, the problem, rising action, solution and climax which, according to Machado (2003), are the four fundamental stages of the main plot of any story. In the theme take_bath, these four plots would contain the following:

Problem: Defy by not doing the rule Rising Action: Experience consequence

Solution: Do the lesson

Climax: $\quad$ Learn the benefit

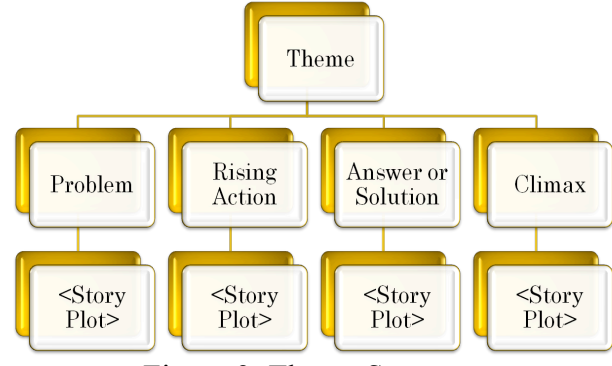

Figure 3. Theme Structure

The theme is executed through the story plot. A story plot represents the major events in the theme and contains at least two author goals (shown in Figure 4) that represent the scenes comprising the event.

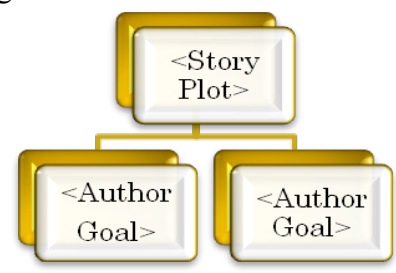

Figure 4. Story Plot Structure

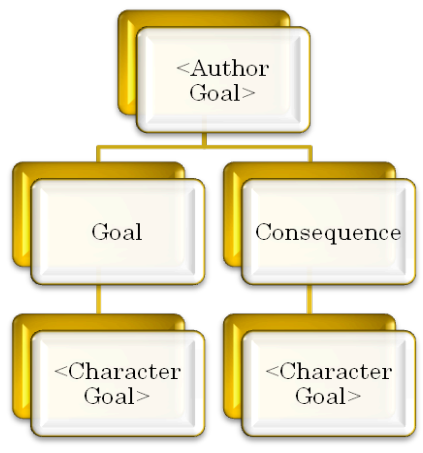

Figure 5. Author Goal Structure

An author goal (Figure 5) is composed of a goal of the scene and the corresponding consequence of the goal to ensure consistency in the scene. Each goal and consequence component of an author goal is in turn filled up by at least one character goal.A character goal (Figure 6) represents the unit of action a character or two characters do in order to depict the goal/consequence of the scene. This design of the character goal is based from the action operators of Uijlings (2006) and can be directly converted to simple declarative sentences.

A character goal has five fields - the action, the agens or doer of the action, the patiens or receiver of the action, the target, and the instrument. One character goal generates one sentence in the story with the agens as the subject, the $a c$ tion as the verb, the patiens as the character or object that undergoes the action verb, the target as the location or object of the action verb, and the instrument as the object used to perform the verb. This design allows all fields to be empty except for action and agens.

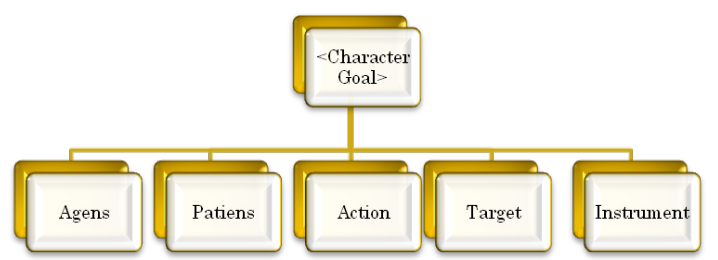

Figure 6. Character Goal Data Structure

The character goal is generic so that it only contains default values for action, agens and $p a$ tiens. During instantiation of a character goal for a particular scene, attribute values are passed to the character goal at the author goal level. For example, given the generic character goal adult tells main character (CGL01) has the following default attributes:

$$
\begin{array}{ll}
\text { action: } & \text { tell } \\
\text { agens: } & \text { adult } \\
\text { patiens: } & \text { main character }
\end{array}
$$

When a scene requires the adult to inform the main character of the lesson, the target attribute would then be assigned with the lesson value to denote that the adult is talking to the main character about the lesson. The invocation of the character goal in the author goal level would be:

\section{CGL01(target:lesson)}

This customizes the character goal to "adult tells the lesson to the main character" to fit the scene.

Parameters for character goal attributes include not only the story variables (i.e. object, lesson, background), but invocations to inner character goals and ontology accesses as well. An inner character goal is a character goal assigned as an attribute of an outer character goal. 
It represents a clause in a sentence and is usually assigned as a value of the target attribute in the outer character goal, for example:

\section{CGL03(target:CGL05(target:lesson))}

Picture Books would interpret the inner character goal "main character is not doing the lesson (CGL05)" first before appending it to the outer character goal "secondary character told the adult character (CGL03)", resulting in the following sentence specification:

secondary character tells an adult that the main character is not doing the lesson

The instrument attribute in a character goal may have the following value which would trigger an ontology search for a concept:

onto $<$ semantic_category $>(\%$ object $\%)$

The <semantic category> constrains the search coverage to concepts that are directly connected to the given input concept (\%object\%). For example, ontoSpatial(play) triggers a search for all concepts connected to play within the spatial semantic category (e.g., "locationOf" and "oftenNear" semantic relationships).

Character goals can also be created dynamically during runtime and are used to increase the length and the variation of the generated story. Dynamic character goals necessitate the search for relationship paths in the ontology. Two input concepts, the source and destination concepts, and the semantic category that constrains the search coverage, are needed when searching for relationship paths.

Consider the following attribute value of a character goal:

ontoEvent(break object, punishment)

This denotes that a search for a series of semantic relationships within the Event semantic category must be performed to relate break object to punishment. The resulting path is shown in Figure 7.

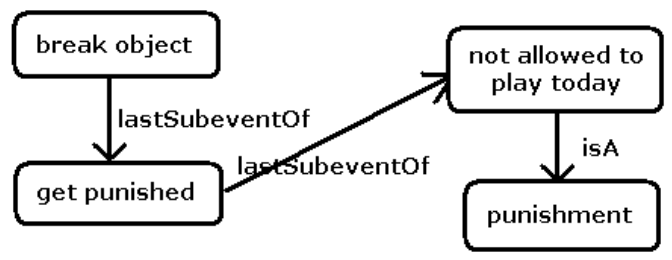

Figure 7. Sample Ontology Path Search Result

Each relationship in the resulting path, excluding is A (hypernym) and conceptuallyRelatedTo relationships, is mapped to a dynamic char- acter goal. For example, in the first lastSubeventOf relationship in Figure 7, the second concept ("get punished") is assigned to the action attribute of the dynamic character goal, resulting in the abstract output sentence specifications "Main character gets punished" and "Main character is not allowed to play today".

\subsection{Planning Process}

The Theme Planner selects a theme by matching the input objects against the applicable objects of the candidate themes for a specified background. When the theme with the highest score has been determined, the Plot Planner instantiates the story plots of the chosen theme, beginning with the problem, followed by the rising action, then the solution and finally the climax. Given the theme eat_on time with the following corresponding story plots:

eat_on_time(defy_break_rule, experience consequence, inform_lesson, realize_benefit) ${ }^{1}$

the first (problem) story plot, defy_break_rule, suggests that the main character will disobey an adult character's rule/instruction. Executing this plot entails executing the author goals within it.

$$
\begin{gathered}
\text { defy_break_rule(inform_character_rule, } \\
\text { ignore_rule) }
\end{gathered}
$$

Then the first author goal, inform_character rule, suggesting that the adult character informs the main character of the rule first, is executed:

\section{inform_character_rule(adult_talk_character,} character_not_want)

Finally, the character goals in the author goal, adult_talk_character and character_not_want, are executed. Notice that it is only in the character goal level that instantiation of characters and objects are made.

$$
\begin{aligned}
& \text { adult_talk_character("tell”, "Mommy Audrey", } \\
& \text { "Simon”, "eat on time”, null) }{ }^{2} \\
& \text { character_not_want("not want”, "Simon”, null, } \\
& \text { "eat on time”, null) }
\end{aligned}
$$

These phrase specifications of character goals will be forwarded later to the surface realizer for translation to simple sentences to generate the text "Mommy Audrey told Simon to eat on time." and "Simon did not want to eat on time.", respectively.

\footnotetext{
${ }^{1}$ Theme format:

Theme(problem, rising action, solution, climax)

${ }^{2}$ Character Goal format:
}

CG(action, agens, patiens, target, instrument) 
The planning process continues by backtracking to the first story plot and iterating until it exhausts all the theme's story plots. A depth-first tree traversal algorithm is employed to recursively traverse the theme tree. An excerpt from the theme eat_on time, starting from the story plot defy_break_rule, and its tree traversal, is shown in Figure 8.

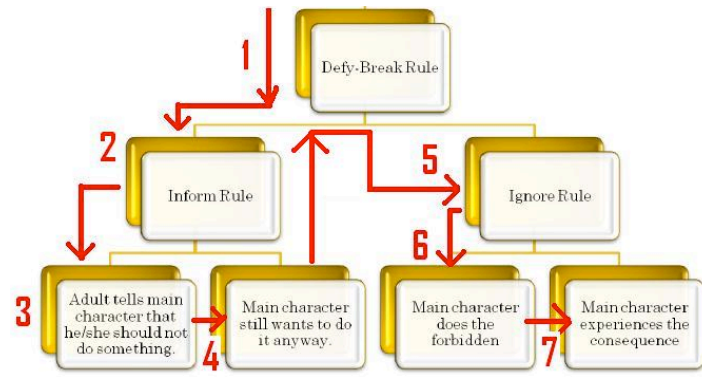

Figure 8. Tree Traversal for the Problem "Defy Break Rule" of the "Eat_On_Time" Theme

\subsection{Planning for Linguistic Variation}

Sentences generated by Picture Books vary in length and word complexity according to the user's age. The variation in length is handled by specificity character goals (SCG) that are appended as supporting details to their respective character goals. SCGs are designed so that their existence will give more detail to the preceding character goal while ensuring consistency, and that their non-existence will still make the story complete. SCG is not executed when the user is four years old, one SCG is executed when the user is five years old, and all of SCGs are executed when the user is six years old.

\begin{tabular}{|l|l|}
\hline Age & Story Excerpt \\
\hline Four & $\begin{array}{l}\text { Ellen was scared. She was sad. } \\
\text { Teacher Sara saw that Ellen was crying. } \\
\text { Teacher Sara told Ellen to be brave. }\end{array}$ \\
\hline Five & $\begin{array}{l}\text { Ellen was scared. She was lonely. } \\
\text { Teacher Sara saw that Ellen was crying. } \\
\text { Teacher Sara asked Ellen if everything } \\
\text { was okay. She told her to be brave. }\end{array}$ \\
\hline Six & $\begin{array}{l}\text { Ellen was scared. She was } \text { upset. } \\
\text { Teacher Sara saw that she was crying. } \\
\text { Teacher Sara asked Ellen if everything } \\
\text { was okay. Ellen told teacher Sara that she } \\
\frac{\text { was scared. }}{\text { Teacher Sara told Ellen to be brave. }}\end{array}$ \\
\hline
\end{tabular}

Table 2. Excerpts of Stories with Linguistic Variation

Word complexity is addressed by storing different synonymous lexical items in the lexicon. The appropriateness of the lexical items to the target age group was verified by child educators. Table 2 contains excerpts from Picture Books' stories for various ages, with boldfaced italics showing lexical variation, while underlined sentences are the executed SCGs. Additional sentences and lexical items can be generated by encoding more SCGs into the plan library and the ontology, respectively.

\section{Results and Analysis}

The knowledge base of Picture Books currently contains 9 backgrounds, 11 themes, 40 characters, 37 objects, 77 author goals, and 61 character goals. The lexicon has been populated with 419 words appropriate for the target age group, while the ontology has 240 concepts and 369 semantic relationships. 15 exemplary stories generated by the system, consisting of five themes that vary per age $(4,5$, and 6$)$, were selected. Two child educators and a linguist manually validated the appropriateness of the content and the linguistic correctness of the 15 stories, respectively.

Each story was rated per criterion from 1 to 4 , with 4 being the highest. A rate of 4 means that the criterion is completely present in the story, 3 means that the criterion is present but incomplete, 2 means the criterion is partially present, and 1 means the criterion is not present.

\subsection{Evaluating the Story Content}

The evaluation on the story patterns' role in ensuring that the story goal is met is shown in Table 3 , while the evaluation of the semantic ontology's role in supplying domain knowledge to the story is shown in Table 4.

The evaluators gave the stories high ratings in terms of plot completeness, validating that the stories have all the essential story elements (problem, rising action, the solution and the climax to the problem) as well as the introduction of the time and setting of the story. This was attributed to the plan library structure consisting of themes, plots, author goals, and character goals. Because of this coherency in the plot, the understandability criterion received a high average score of 3.47 .

\begin{tabular}{|l|r|}
\hline Criteria & Average \\
\hline Story is understandable & 3.47 \\
\hline $\begin{array}{l}\text { The settings of the story were de- } \\
\text { scribed }\end{array}$ & 3.86 \\
\hline $\begin{array}{l}\text { The characters in the story were } \\
\text { described }\end{array}$ & 3.67 \\
\hline Objects are present in the story & 4.00 \\
\hline General Average & $\mathbf{3 . 7 5}$ \\
\hline
\end{tabular}

Table 3. Evaluation on the Story Patterns 


\begin{tabular}{|l|r|}
\hline Criteria & Average \\
\hline Sentences are coherent & 2.67 \\
\hline The story has transition & 3.47 \\
\hline $\begin{array}{l}\text { The actions of the characters make } \\
\text { sense }\end{array}$ & 3.93 \\
\hline Story is appropriate to target age & 3.80 \\
\hline Objects in the story were described & 2.80 \\
\hline General Average & $\mathbf{3 . 3 3}$ \\
\hline
\end{tabular}

Table 4. Evaluation of the Semantic Ontology

Evaluations on the effectiveness of the semantic ontology, on the other hand, produced varying scores. The coherency of the sentences received a low average score of 2.67 , because as seen in the excerpt below, a sentence (such as the underlined text) depicting that Ellen the elephant did something to show she is trying to be brave was missing in the generated story.

She wanted to be brave. Ellen was brave. She wanted to play with others. She bravely introduced herself. Ellen made friends.

Since the ontology did not contain any other information of what to do in order to be brave, the story content planner did not place any detail describing the action of the main character depicting her attempt to be brave. This can be remedied by adding more semantic relationships (that can be converted to sentences) between concepts.

The excerpt from the generated story "Rizzy the Rabbit Learns to be Honest" below also affected the coherency criterion when the second character, Denise the dog, suddenly appeared in the middle of the story when she could have been introduced with the first character, Rizzy the rabbit, at the start of the story.

The evening was warm. Rizzy the rabbit was in the dining room. She played near a lamp. Rizzy broke the lamp. She was scared. Mommy Francine saw that the lamp was broken. Rizzy told Mommy Francine that Denise broke the lamp.

The criterion regarding story transition pertains to the ease of transition of events that can be attributed to the path of relationships retrieved from the semantic ontology, which more often than not, introduce event transitions including character actions.

The criterion on appropriateness of the story content to the target age received a high average score of 3.80 mainly because the semantic ontology has been populated with concepts specifically for the target age group.
While the presence of objects in the stories received a high score of 4.00 (see Table 3 ), the description of objects received a low score of 2.80 (see Table 4), because although objects are present in the stories, as in "She played near a lamp.", they were not described, i.e., "breakable lamp". This was remedied by adding object descriptions in the ontology. However, the selection of which description to be used was currently not dictated by the story theme, nor was the description subsequently used to direct the sequences of events in the rest of the story.

\subsection{Evaluating the Linguistic Aspect}

Table 5 summarizes the evaluation on the grammatical aspect of the generated stories. Since mostly simple sentences are generated, they are grammatically correct and coherent.

\begin{tabular}{|l|r|}
\hline \multicolumn{1}{|c|}{ CRITERIA } & AVG \\
\hline Sentences are grammatically correct & 3.20 \\
\hline Sentences are coherent & 3.60 \\
\hline Pronouns are used correctly & 3.45 \\
\hline Articles are used correctly & 3.20 \\
\hline The story has transition & 3.00 \\
\hline General Average & $\mathbf{3 . 2 9}$ \\
\hline
\end{tabular}

Table 5. Evaluation on Grammar Correctness

Most of the generated stories contain correct usage of pronouns, except for sentences such as "Teacher Sara told Ellen that Ellen should be brave", where the second reference to "Ellen" should be replaced with the pronoun "she". The evaluators, however, noted the lack of possessive pronouns in the text below (the underlined words are the identified missing pronouns).

Porky wanted to play. He played with his toys. His toys were scattered.

There are also occurrences of missing articles, as shown in the excerpt below (the underlined words are the missing articles).

He played near a glass of water. Simon broke the glass of water. He was scared. Daddy Gary saw that the glass of water was broken.

The generated stories received an average score of 3.0 for the transition criterion due to missing transitional devices, as shown in the excerpt below (the underlined words are the missing transitional devices).

He apologized to her. Mommy Patricia then helped Porky to clean up. Soon he found the lost toys. 


\section{Conclusion and Recommendations}

The design of Picture Books' plan library, combined with planning operators in the form of story plots and character goals, demonstrated that grammatically correct and coherent stories for children aged 4 to 6 can be generated from a given set of predefined pictures, provided that the appropriate domain knowledge is present. The semantic ontology whose design was adapted from ConceptNet provided the domain knowledge that supplies factual information to the story. The theme structure and its components model the way humans perform storytelling and also ensured that the generated stories will contain the four basic elements of problem, rising action, solution, and climax. The separation of the plan library which dictates the story patterns from the semantic ontology gives flexibility to Picture Books such that it could easily be adapted to generate other story domain.

Currently, Picture Books generates stories with simple declarative sentences. A future improvement of the system is to extend the design of the character goal to generate stories with dialogues. Although common animals that young children can relate with have been used as the main characters of the story, their traits, such as loyalty, bravery, and kindness, have not been considered in determining the flow of the story. A similar claim can be made for describing the objects used in the story, such that a story promoting the value of "being thrifty" can use the "expensive lamp" as an object, while the value of "being careful" can use the "breakable lamp" instead. These are aspects of storytelling that can be investigated in a future work.

From a linguistic perspective, the current implementation of Picture Books can generate pronouns and articles, as well as a transitional device at the last sentence in the story. However, possessive pronouns, the consistent use of articles, and the generation of appropriate transitional devices in several parts of the story should be further explored. The design of the character goals can be extended by adding an attribute indicating a connection or the passage of time between sentences. Rhetorical Structure Theory (Mann and Thompson, 1988) can also be applied to author goals and character goals for an effective discourse structure that would provide a smoother story flow.

\section{References}

Charles B. Callaway, and James C. Lester. 2002. Narrative Prose Generation. Artificial Intelligence, 139(2): 213-252.

Charles B. Callaway. 2000. Narrative Prose Generation. PhD thesis, North Carolina State University, Raleigh, NC.

Robert Dale, and Ehud Reiter. 2000. Building Natural Language Generation Systems. Cambridge: Cambridge University Press.

Marjorie Fields, Lois Groth, and Katherine Spangler. 2003. Let's Begin Reading Right: A Developmental Approach to Emergent Literacy, 5/E. Prentice Hall.

Hugo Liu, and Push Singh. 2004a. Commonsense Reasoning in and over Natural Language. In Proceedings of the 8th International Conference on Knowledge-Based Intelligent Information and Engineering Systems, 293-306, Wellington, New Zealand. Springer Berlin.

Hugo Liu, and Push Singh, 2004b. ConceptNet - A Practical Commonsense Reasoning Tool-Kit. BT Technology Journal, 22(4): 211-226. Springer Netherlands.

Hugo Liu, and Push Singh. 2002. Makebelieve: Using Commonsense Knowledge to Generate Stories. In Proceedings of the Eighteenth National Conference on Artificial Intelligence, 957-958, Edmonton, A1berta, Canada. AAAI Press.

Birte Loenneker. 2005. Narratological Knowledge for Natural Language Generation. In Proceedings of the Tenth European Workshop on Natural Language Generation, 91-100, Aberdeen, Scotland.

Jeanne Machado. 2003. Storytelling. In Early Childhood Experiences in Language Arts: Emerging Literacy, 304-319. Clifton Park, N.Y. Thomson/Delmar Learning.

William C. Mann, Sandra A. Thompson. 1988. Rhetorical Structure Theory: Toward a Functional Theory of Text Organization. Text, 8(3), 243-281.

James Meehan. 1977. TALE-SPIN, An Interactive Program that Writes Stories. Proceedings of the 5th International Joint Conference on Artificial Intelligence, 91-98. Cambridge, M.A.

Scott R. Turner. 1992. Minstrel: A Computer Model of Creativity and Storytelling. Los Angeles, California: University of California.

Jasper Uijlings. 2006. Designing a Virtual Environment for Story Generation. MSc Thesis, University of Amsterdam, Amsterdam.

Chris Venour, Ehud Reiter. 2008. A Tutorial for Simplenlg (version 3.7) http://www.csd.abdn.ac.uk/ ereiter/simplenlg/

WordNet: A Lexical Database for the English Language. Princeton University, New Jersey, 2006. 